\title{
Segmentation and Measurement of Medical Image Quality Using K-means Clustering Algorithm
}

\author{
Ahmed Mohamed Ali Karrar, Jun Sun \\ School of Internet of Things \& Engineering, Jiangnan University, Wuxi, China \\ Email address: \\ karrar@yahoo.com (A. M. A. Karrar), junsun_wx@hotmail.com (J. Sun) \\ To cite this article: \\ Ahmed Mohamed Ali Karrar, Jun Sun. Segmentation and Measurement of Medical Image Quality Using K-means Clustering Algorithm. \\ American Journal of Neural Networks and Applications. Vol. 5, No. 1, 2019, pp. 36-44. doi: 10.11648/j.ajnna.20190501.16
}

Received: April 16, 2019; Accepted: June 27, 2019; Published: July 16, 2019

\begin{abstract}
In this paper we have segmented an image by using a k-clustering algorithm, using the Gaussian Mixture Model cluster to generate the initial centroid. Many types of research have been done in the area of image segmentation using clustering especially medical images, these techniques help medical scientists in the diagnosis of diseases thereby to cure this diseases $\mathrm{K}$-means clustering algorithm one of these techniques, it is an unsupervised algorithm and it is used to segment the interest area from the background. We used also partial contrast stretching to improve the quality of the original image. And the final segmented result is comparing with the k-means clustering algorithm and we can conclude that the proposed clustering algorithm has better segmentation. Finally, MSE and PSNR are checked and discovered that they have small and large value respective, which are the condition for good image segmentation quality. And comparison for MSE and PSNR are done for the proposed method and classical K-means algorithm and it is found that the proposed method has better performance result.
\end{abstract}

Keywords: Image Segmentation, K-means Clustering, Partial Contrast Stretching, Gaussian Mixture Models

\section{Introduction}

Image segmentation is a very important technology for image processing. There are many applications whether on the synthesis of the objects or computer graphic images require precise segmentation. With the consideration of the characteristics of each object composing images in MPEG4, object-based segmentation cannot be ignored.

Image segmentation is that the method of partitioning images into multiple segments (sets of pixels, also known as super-pixels). The goal of segmentation is to simplify and change the representation of an image into separate regions that are a lot of meaningful and easier to analyze. [1, 2] Image segmentation is typically used to find objects and boundaries (lines, curves) in images. More exactly, image segmentation is that the method of assigning a label to each pixel in an image such that pixels with the same label share certain characteristics.

Image segmentation is a valuable tool in many fields including health care, image processing, traffic image, pattern recognition etc. There square measure completely different techniques for image segmentation like threshold based, edge-based, cluster-based, neural network based1. From a different technique, one of the most efficient methods is the clustering method. Again, there are different types of Method and a subtractive clustering method. One of the most used clustering algorithms is $\mathrm{k}$-means clustering. It is simple and computationally faster than the hierarchical clustering.

Image segmentation becomes one of an important tool in the medical area where it is used to extract or region of interest from the background. When applied to a stack of images, typical in medical imaging, the resulting contours after image segmentation can be used to create 3D reconstructions with the help of interpolation algorithms. So medical images are segmented using a different technique and process outputs are used for further analysis in medical. But medical images in their raw form are represented by the arrays of numbers in the computer, with the number indicating the values of relevant physical quantities that show the contrast between different types of body parts. Processing and analysis of medical images square measure helpful in reworking raw images into a quantitative symbolic type, in extracting purposeful qualitative data to help identification and in the integration of complementary data from multiple imaging modalities. And one of the fundamental problems in the medical analysis is the image segmentation which identifies the boundaries of objects 
such as organs or abnormal region in images data from multiple imaging modalities.

The result of image segmentation may be a set of segments that collectively cover the entire image or a set of contours extracted from the image. Each of the pixels in a region is similar with respect to some characteristic or computed property, like color, intensity, or texture. Adjacent regions square measure considerably totally different with regard to a similar characteristic(s). [1]

Results from the segmentation build it a potential for shape analysis, police investigation volume amendment, and creating a certain irradiation treatment plant.

\section{Classification of Image Segmentation Techniques}

There are many existing techniques that area unit used for image segmentation. These techniques have their own importance. These all techniques will be approached from two basic approaches of segmentation i.e. region based or edge-based approaches. Every technique will be applied to totally different images to perform needed segmentation. These techniques can also be classified into three classes [3-4].

\section{A. Structural Segmentation Techniques}

The structural techniques are those techniques of image segmentation that depends upon the information of the structure of the required portion of the image i.e. the required region which is to be segmented.

\section{B. Stochastic Segmentation Techniques}

The Stochastic techniques are those techniques of the image segmentation that works on the separate pixel values of the image instead of the structural info of region.

\section{Hybrid Techniques}

The hybrid techniques area unit those techniques of the image segmentation that uses the concepts of higher than techniques these uses discrete pixel and structural info along.

In this paper, the various techniques of segmentation are discussed and compared. The mathematical description is avoided for simplicity. The popular techniques used for image segmentation are thresholding technique, edge detection-based techniques, region-based techniques, clustering based techniques, watershed-based techniques, partial differential equation based mostly and artificial neural network-based techniques etc.

These all techniques are different from each other with respect to the method used by these for segmentation.

The classification is given in Figure 1.

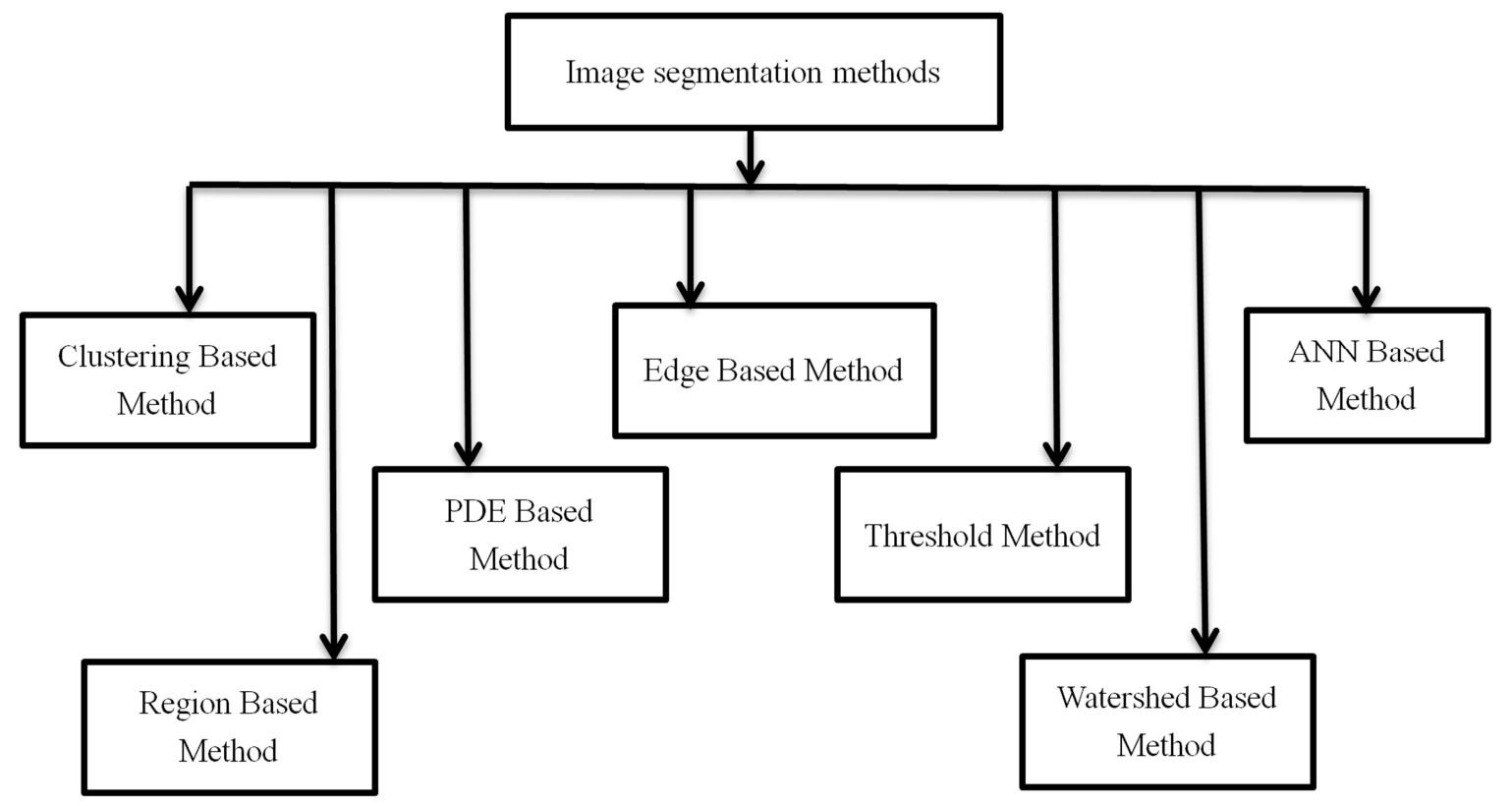

Figure 1. Image Segmention techniques.

\section{Literature Review of Image Segmentation}

Many works done in the area of image segmentation by using different techniques. And many are done supported completely different application of image segmentation. $\mathrm{K}$ -means algorithm is the one of the simplest clustering algorithms and there are many methods implemented so far with different method to initialize the center and many researchers are also making an attempt to supply new techniques that are an additional economical than the present strategies, and shows better segmented result. Some of the present recent works are mentioned here.

Fernando C. Monteiro [5] they gave us a new image segmentation technique comprises of edge and region-based information with the help of spectral technique and morphological algorithm of watershed. they reduce the noise from image using bilateral filter as a pre-processing step, and they region merging is used to perform preliminary segmentation, region similarity is generated and then graph based region grouping is perform using Multi-class Normalized Cut method [6]. Berkley segmentation dataset is use as a dataset. They compare the technique with mean shift; 
multi-scale graph based mostly segmentation, and JSEG. It is found that proposed technique has outperformed other methods and produce good results.

R. V. Patil said that if the number of clusters is estimated inaccurate manner, K-means image segmentation will provide better results [7]. They gave a new method based on edge detection to estimate the number of clusters. Phase congruency is used to detect the edges. Then these edges are used to find clusters. Threshold and Euclidean distance are used in order to make clusters. K-means issued to find the final segmentation of the image. MATLAB is used to implement the proposed technique. Experiments are performed on nine different images and results show that the number of clusters is accurate and optimal.

Weihong Cui Yi Zhang [8] projected an edge-based auto threshold choose the method to get multi-scale image segmentation. Band weight and NDVI (Normalized Difference Vegetation Index) is used to calculate edge weight. MST and Edge-based Threshold method are used to perform image segmentation. Experiments are performed on multi-scale resolution images, i.e., Quick-bird multispectral images. Results have shown that their method maintains the object information and keep object boundaries while segment the image.

Anna Fabijańska introduced a new method uses Variance Filter for edge detection in the image segmentation process [9]. Their method found the edge position using Variance Filter. Sobel Gradient filter with K-means is used to extract the edges and compared with the proposed method.

Mohammed J. Islam found that Computer Vision is a better solution for real time inspection of capsules in pharmaceutical industry [10]. Author has developed a system for quality scrutiny victimization edge based mostly image segmentation methods. They used Sobel Edge Detector so as to observe edges with noise-suppression property. After edge detection, Otsu Thresholding method is used for localization of background and foreground pixels. Experiments square measure conducted and results square measure compared with NN-based segmentation technique building Visual $\mathrm{C}++$. Results outperform NN method on the basis of accuracy and processing time difference of $10 \mathrm{~ms}$.

Liu Yucheng used a new fuzzy morphological based fusion image segmentation algorithm [11]. Algorithm has used morphological closing and opening operations to smooth the image and then perform the gradient operations on the resultant image [12]. Found that fusion approach solves the problem of over-segmentation of Watershed algorithm after compare the proposed fusion with Watershed algorithm [13] and Prewitt techniques. It conjointly saves the data details of image and improves the speed.

Syoji Kobashi presented a fuzzy object model to segment the cerebral parenchyma region of newborn brain MRI image and scale based fuzzy connected image segmentation [14]. Foreground region is separated in the first step, correction of MRI intensity in-homogeneity is performed next, and then scale-base Fuzzy Object Model (FOM) is applied on the resultant image. Results are evaluated on the basis of Fast
Positive Volume Fraction (FPVF) and Fast Negative Volume Fraction (FVNF). Results from the experiment have given that FOM (Fuzzy object model) has attained minimum FPVF and FVNF values.

Refik Samet used a new Fuzzy Rule-based image segmentation technique to segment the rock thin segment images [15]. They use RGB image of rock thin segment (input) and give segmented mineral image (output). Fuzzy C Means is also applied on rock thin images and results are compared of both methods. Firstly, the user can take a sample image from minerals; features are distinguished on the basis of red, green and blue components of the image. The membership function is defined for each component using Fuzzy rules. Strong and weak points are defined, whereas strong points are considered as seed points and weak points become their members. Results have given that the proposed method is better than the FCM algorithm.

Muhammad Rizwan Khokher used a Fuzzy Rule-based system and Graph Cuts [16]. Authors have firstly segmented the grayscale, color, and texture images using Graph Cuts. Weights are appointed to the features of the image using Fuzzy Rules. In their project algorithm works by extracting the options of the image, calculate the constants using fuzzy rules, calculate the weighted average of constants to find the similarity matrix, partition the graph using Normalized Graph Cut technique [17], and finally get the segmented image from the partitioned graph. Berkley database is used to evaluate the algorithm. Simulation is performed in Matlab and C language. Results are evaluated on the basis of Mean, PPV value and Standard Deviation.

Jinsheng Xiao [18] used a new non-linear discontinue partial differential equation (PDE) that models the level set method of gray images [18]. A discrete method is also proposed to find a numerical solution and to implement the filter. Non-Linear discontinue PDE formula is applied to the image of cameramen using MATLAB. Results have shown that image edges and boundaries are remained blurred and can be shifted by using the Close operator. the information saved by using the proposed scheme.

Fengchun Zhang used a variation model using 4th order PDE with 2nd order PDE for finger vein image de-noising [19]. Midpoint Threshold segmentation technique is employed to extract the region of interest accurately. 4th order PDE has reduced the noise fine, whereas 2nd order PDE has approximated the boundaries effectively. It can be observed from experiments that PSNR value of the proposed method is increased by two $\mathrm{dB}$. The method is compared with threshold-based segmentation algorithm and it is found that the technique has segment the real finger vein image accurately.

Chun Yuan used a new segmentation model for color images [20]. Their model is based on the Geodesic Active Contour (GAC) model. But GAC is only restricted to grayscale images. Therefore, their model is also an extension of GAC model and known as a color-GAC model. It uses the expression of the Gradient of the color image.

Wencang Zhao proposed a new image segmentation 
algorithm based on textural features and Neural Network to separate the targeted images from the background [21, 22, 23]. Dataset of micro-CT images is used. De-noising filter is used to remove noise from the image as a pre-processing step, Feature extraction is performed next, and then Back Propagation Neural Network is created, and lastly, it modifies the loaded variety of network, and save the output. The proposed algorithm is compared with Thresholding method and Region Growing method. Results have shown that the proposed technique outperforms other methods on the basis of speed and accuracy of segmentation.

Lijun Zhang used a new neural network-based image segmentation system for color images [24]. They combined the Wavelet Decomposition and Self Organizing Map (SOM) to propose a brand-new technique, i.e., SOM-NN. Voting among child pixels selected the parent pixel. After initialization, ANN found the segmentation result that satisfies all levels. Wavelet decomposition is performed to remove noise. Hence wavelet decomposition and SOM-NN are combined to perform segmentation. Results have shown that the method has to reduce noise and produce accurate segmentation.

Shohel Ali Ahmed used the Image Texture Classification technique based on Artificial Neural Networks (ANN) [25]. Firstly, the image is captured and pre-processing is performed, after it, feature extraction is performed, whereas, ANN classifier is used for texture classification, Clustering is performed to separates background from sub-images [26, 27]. Trained ANN combines the input pixels into 2 clusters which give results. It produces the texture classification and segmentation of the image.

Shiping Zhu used a new threshold-based edge detection and image segmentation algorithm [28]. He calculates the threshold of each pixel in the image on the basis of its neighboring pixels. They also find the edges of the image with the help of the proposed algorithm. A threshold of each pixel was set using the histogram. PDF is used to isolate the background and threshold of the image. They implement their algorithm in Visual $\mathrm{C}++$. Results outperform the Canny Operator results because it performs edge detection and segmentation at the same time.

Anping XU used a threshold-based level set approach comprising each threshold-based segmentation and quick march Method (FMM) for medical image segmentation [29, 30]. The result of the de-noising filter is passed to FMM for segmentation purpose with the help of threshold-based level set technique. They implement their method in $\mathrm{VC}++$ and ITK. After the experiment, results have shown that the level set method based on threshold results in clearer, accurate and more perfect segmentation, it improves the speed of segmentation and avoid edge leakage.

Wu Kaihua and Ban Tao have presented a new optimal threshold segmentation method based on entropy criteria and Genetic algorithm in order to improve the image acquisition process in computer vision [31]. The factors taken by them are illumination, light, the reflection of light, CCD exposure time and some properties of the image histogram. They compare their proposed technique with the Otsu algorithm and found that their algorithm is efficient in searching and in finding threshold-based segmentation of an image.

Frank Jiang proposed a new multilevel Threshold-based segmentation technique using PSO and Wavelet mutation. They also proposed a new PSO algorithm which is used in the first two steps of an algorithm [32]. Then the output of PSO is passed to wavelet mutation operations which perform the mutation operation and update the PSO equations after it. This work will generate an optimized threshold and correct segmentation. After comparing their method with HCOCLPSO, they found that it produces an optimal threshold as compared to another method. They claim that their algorithm is best for real-time applications, e.g., error resilient video application in a hostile environment.

D. Barbosa planned a brand-new image segmentation technique that joins the edge and region-based information with a spectral method using Morphological Watershed algorithms [33]. Firstly, noise filter is used with Magnitude Gradient in a pre-processing stage, secondly, pre-segmentation is done using region merging, then region similarity graph is generated and finally, segmentation is performed using Multi Class Normalized Cut. The method is compared with Mean Shift, MNCUT, and JSEG using natural images. The proposed technique overcomes the Spectral Clustering method.

\section{Contrast Enhancement Using Partial Contrast Stretching}

Some images used in medical analysis (e.g. eye images) have their own weaknesses such as low contrast. Therefore, a contrast enhancement technique such as partial spatial starch (PCS) is used to improve image quality and contrast in image [34]. This is done by stretching and pressing. By applying this technique, the pixel range for the minimum value and threshold value will be set to a new pixel range and linearly extends to a wide range of pixels within the new minimum expansion value, and the remaining pixels will face compression.

\section{Gaussian Mixture Models}

Gaussian mixture models (GMM) are used for data clustering. Usually, fitted GMMs cluster by assigning query data points to the variable normal components that maximize the component posterior probability given the data. That is, given a fitted GMM, cluster assigns question data to the component yielding the highest posterior probability.

Very simple and effective. It estimates the number and initial location of the cluster centers. It distributes the data space into a grinding point and computes the potential For each data point base on its distance to the actual data point. The grid point will have high potential value with many data point nearby. And so this grid point with highest potential value will be chosen as a first cluster center. So after selecting 
the first cluster center, we will try to find the second cluster center by calculating the highest potential value in the remaining grid points. As grid points near the first cluster center will reduce its potential value, the next cluster center will be a grid with many data point nearby other than the first cluster center grid point. So this procedure of acquiring a new cluster center and reducing the potential of surrounding grid point repeat until the potential of all grid points falls below a threshold value. We can say this method is one of the simplest and effective methods to find the cluster centers. But with an increase in the dimension of data, its computation complexity grows exponentially. Subtractive clustering algorithm solves the computational method associated with mountain technique It uses data points as the candidates for the cluster center and the computation of this method is proportional to the problem size.

Consider a collection of $\mathrm{n}$ data points: $\mathrm{X}=\{\mathrm{x} 1, \mathrm{x} 2, \mathrm{x} 3 \ldots \mathrm{xn}\}$. Then each point is considered as a potential cluster center. The potential of data point's $\mathrm{xn}$ is defined as:

$$
\left.p(x)=\sum_{k=1}^{k} \pi_{k} \mathcal{N}\left(x \mid \mu_{k}\right), \sum_{k}\right)
$$

Here $\pi_{k} \geq 0$ are the mixing coefficients, with $\sum_{k=1}^{k} \pi_{k}=1$, and $\left(\mu_{k}, \sum_{k}\right)$ are the means and covariances of the component Gaussians.

For a given $\mathrm{K}$, the parameters $\left\{\left(\mu_{k}, \pi_{k}, \sum_{k}\right)\right\}_{k}^{k}=1$ of the model can be fit to the data $\{x(\vec{x})\} \vec{x} \in x$ using maximum-likelihood can be used to select the number of components, $\mathrm{K}$.

A natural approach is then to model the observed feature vector distribution using a mixture of Gaussians model.

After finding the potential of each data points, select the data point with maximum potential as the first cluster Centre. Let us consider $\mathrm{x} 1$ and $\mathrm{p} 1$ as first cluster Centre and its corresponding potential respectively. Then revise the potential of every data point by using the formula given below:

$$
c((X)) \rightarrow=\operatorname{argmax}_{k}\left[\frac{\left.\pi_{k} \mathcal{N}\left(x \mid \mu_{k}\right), \Sigma_{k}\right)}{p(x)}\right]
$$

$\mathrm{rb}$ is the hyper sphere penalty radius in data space and it is a positive constant. Here an amount of potential is subtracted from each data point as a function of distance from the first cluster center. The data points near the first cluster center will have greatly reduced potential, and therefore it has less chance to select as next cluster center. After calculating the revise potential of every data points, find the next highest potential as the next cluster center. these processes continue until a sufficient number of cluster Centre are obtained.

\section{K-Means Clustering Algorithm}

Data clustering is one of the methods widely applied in image segmentation and statistic. The main concept of data clustering is to use the centroid to represent each cluster and base on the similarity with the centroid of the cluster to classify. According to the characteristics of the clustering algorithm, we can roughly divide into "hierarchical" and "partitional" clustering. Except for these two classes, the mean shift algorithm is part of data clustering, too, and its concept is based on density estimation.

Clustering is a technique to divide a set of data into a specific number of groups. It's one of the popular methods is $\mathrm{k}$-means clustering. In k-means clustering, it partitions a collection of data into a $\mathrm{k}$ number group of data. It classifies a given set of data into $\mathrm{k}$ number of disjoint clusters. $\mathrm{K}$ -means algorithm consists of two separate phases. In the first phase it calculates the $\mathrm{k}$ centroid and in the second phase, it takes each point to the cluster which has the nearest centroid from the respective data point. There are different methods to define the distance of the nearest centroid and one of the most used methods is Euclidean distance. Once the grouping is done it recalculate the new centroid of each cluster and based on that centroid, a new Euclidean distance is calculated between each center and each data point and assigns the points in the cluster that have minimum Euclidean distance. Each cluster in the partition is outlined by its member objects and by its centroid. The centroid for each cluster is the point to that the sum of distances from all the objects in that cluster is minimized. $\mathrm{K}$-means is iterative in which it minimizes the sum of distances from each object to its cluster centroid, over all clusters.

Let us consider an image with resolution of $x \times y$ and the image have to be cluster into $k$ number of clusters. Let $p(x, y)$ be an input pixel to be cluster and ck be the cluster centers. The algorithm for k-means 13 clustering is following as:

1. Initialize number of cluster $\mathrm{k}$ and Centre.

2. For each pixel of an image, calculate the Euclidean distance $d$, between the center and each pixel of an image using the relation given below.

$$
d=\|p(x, y)-c K\|
$$

3. Assign all the pixels to the nearest center based on distance $\mathrm{d}$.

4. After all, pixels have been assigned; recalculate the new position of the center using the relation given below.

$$
c k=\frac{1}{k} \sum_{y=c_{k}} \sum_{y=c_{k}} p(x, y)
$$

5. Repeat the process until it satisfies the tolerance or error value.

6. Reshape the cluster pixels into image.

Although k-means has the great advantage of being easy to implement, it has some drawbacks. The quality of the final clustering results is depending on the arbitrary selection of initial centroid. So, if the initial centroid is randomly chosen, it will get different result for different initial centers. So, the initial center will be carefully chosen so that we to consider while designing the $\mathrm{K}$-means clustering. It relies on the number of data elements, number of clusters and number of iterations.

\section{Proposed Algorithm}

The proposed algorithm consists of partial contrast stretching, Gaussian mixture model, k-means clustering. 
Mostly the medical images that are used for segmentation have low contrast. So, contrast stretching is used to improve the standard of the image. After improving the quality of the image, the Gaussian mixture model is used to generate the centers, based on the potential value of the image. A number of the center is generated based on the number of cluster $\mathrm{k}$. This center is used as an initial center in the k-means algorithm. the image is segmented into k number of clusters, Using the k-means algorithm, After the segmentation of the image, the image can still contain some unwanted region or noise. The proposed algorithm is followed as below (Figure 2).

1. Load the image to be segmented.

2. Apply partial contrast stretching. Initialize a number of clusters, $\mathrm{k}$.

3. Calculate the potential for every pixel value of the image. using equation (1).

4. Find maximum potential in step 3 and set that point to be a first center cluster and its corresponding potential as maximum potential.

5. To update the potential value of other remaining pixels based on the first cluster center Use equation (2).

6. Again find the maximum potential in step 4 and let that point be the second point.

7. Continue the process until it finds the $\mathrm{k}$ number of clusters.

8. Used $\mathrm{k}$ center as the initial center in the $\mathrm{k}$-means clustering algorithm.

9. Get the Euclidean distance of each centroid from every pixel of the image using the relation (3).

10. Assign the pixel with minimum distance with respect to centroid to its respective cluster of the centroid.

11. Using equation (4) to recalculate the new central location.

12. Repeat the steps 10-12.

13. Reshape the cluster into the image.

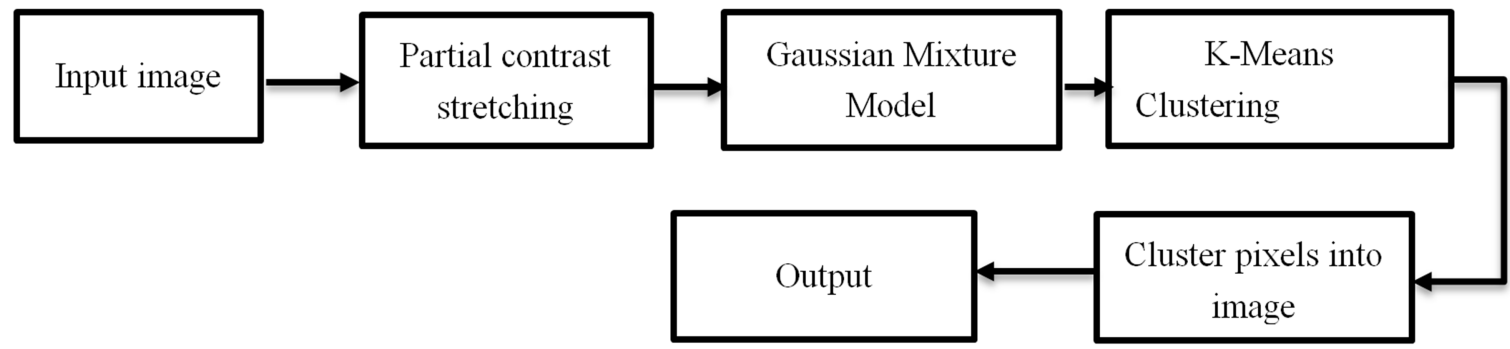

Figure 2. Block Diagram of the proposed Algorithm.

IMG1

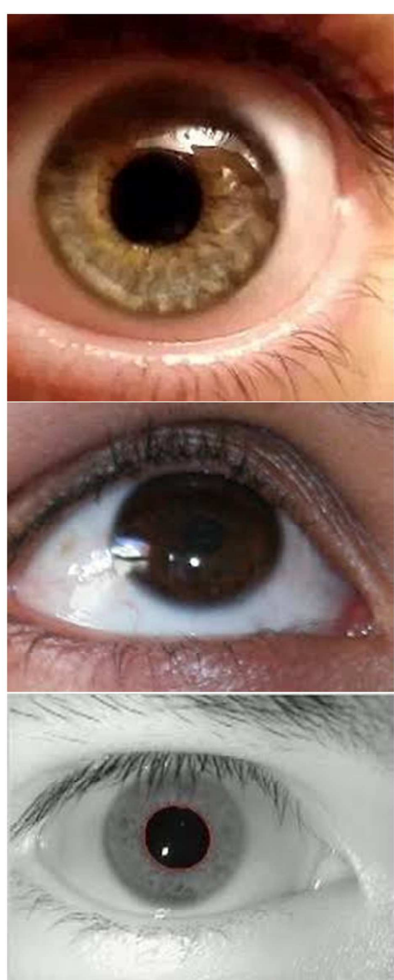

Original image
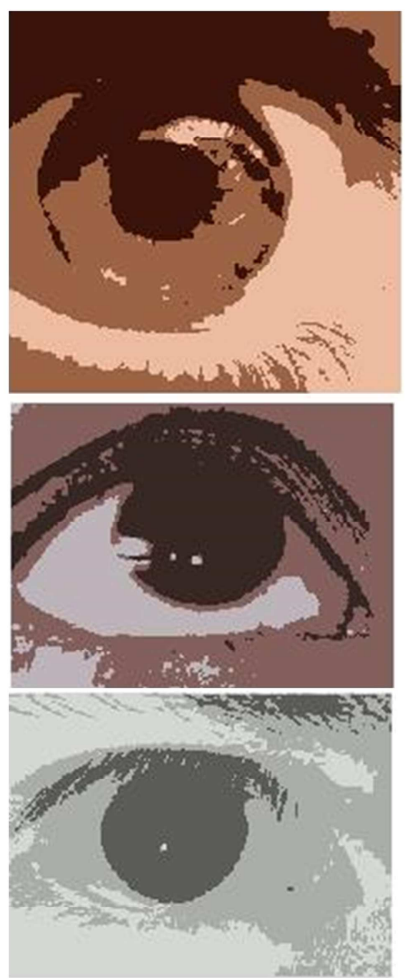

K-means algorithm

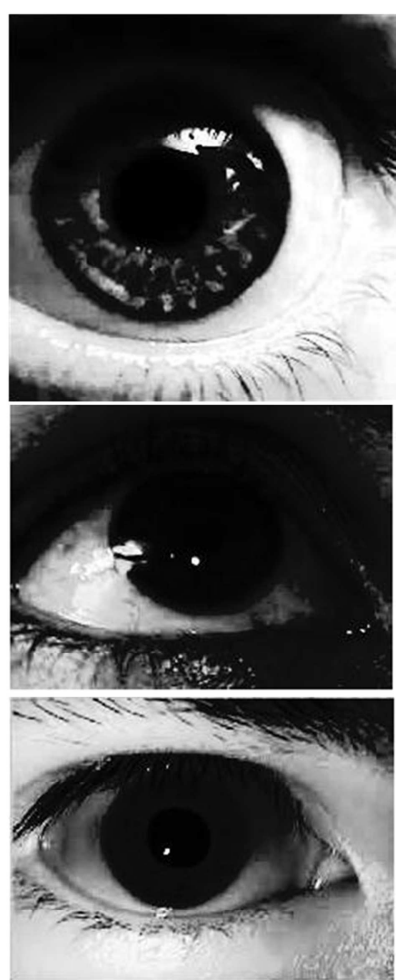

Proposed algorithm

Figure 3. Original image, K-means algorithm, proposed algorithm. 


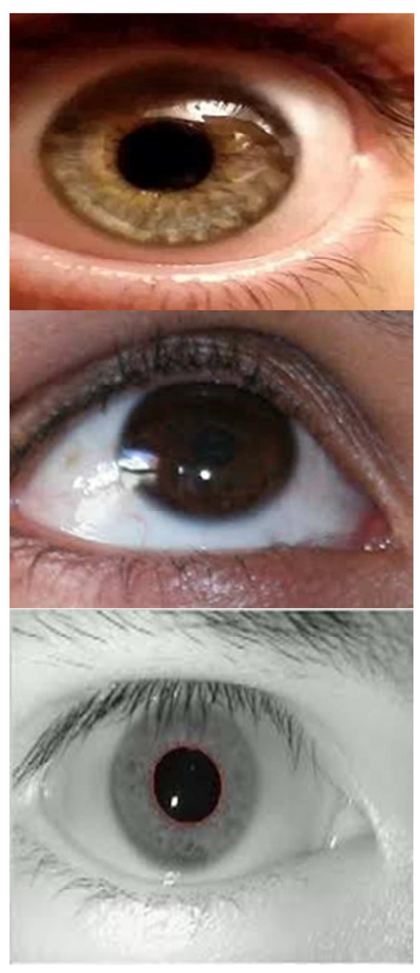

Original image
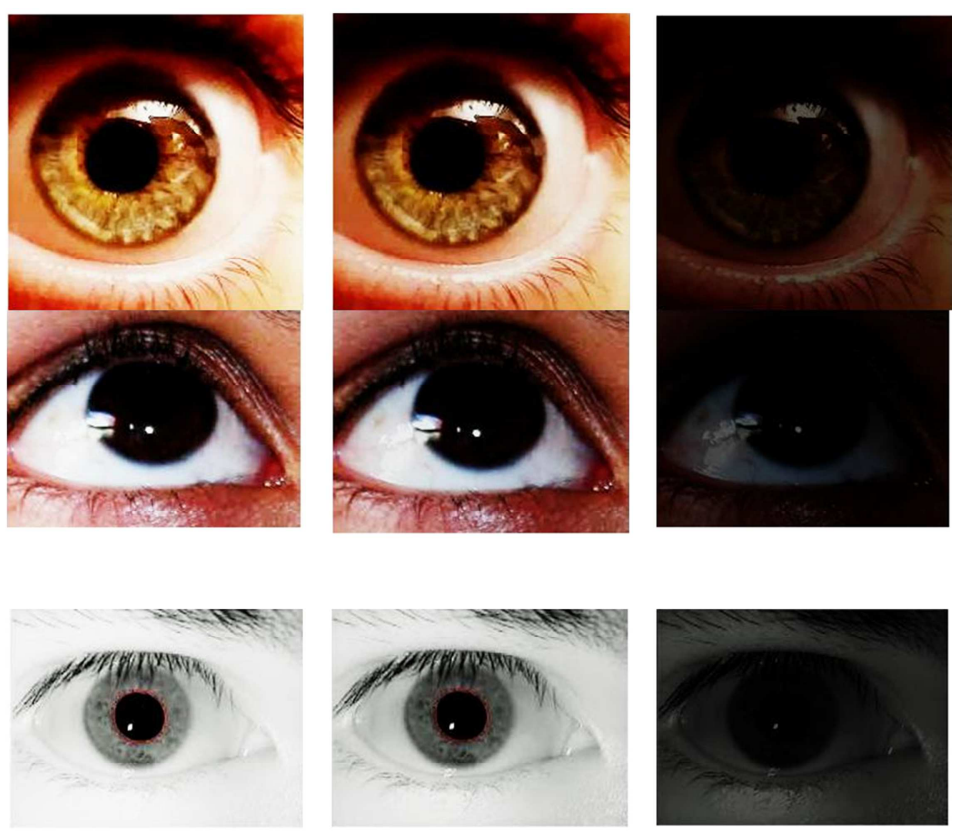

$\mathbf{r b}=\mathbf{0 . 4}$

$\mathbf{r b}=\mathbf{0 . 5}$

$\mathbf{r b}=1.2$

Figure 4. Original image; Proposed algorithm with $r b=0.4$; Proposed algorithm with $r b=0.5$; Proposed algorithm with $r b=1.2$.

\section{Results}

I used different medical images for the analysis. In medical image analysis, mostly the infected areas or area of interest are segmented from the background. We used infected Eye cell-like short and long-sighted eye infected Eye cell for the analysis. Matlab is used to implement the proposed algorithm. We compare the result of $\mathrm{k}$ - means algorithm with the proposed algorithm and it is shown in Figure 3.

In the Gaussian Mixture Model, we can find the number of cluster $\mathrm{k}$. But instead of finding the number of clusters we have given the user-defined number of cluster $\mathrm{k}$ based on the type of the image. Here we have taken the number of clusters, $\mathrm{k}=3$. Now in the Gaussian Mixture Model cluster, we can tune the output image by using the difference value of hyper sphere cluster radius, ra and hyper sphere penalty radius, rb. We know that ra defines the neighborhood distance, so changing this constant will affect the output. In the Figure 4. We got output images for different value

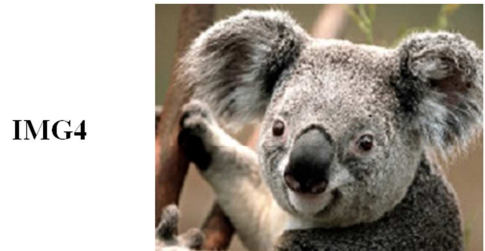

Original Image

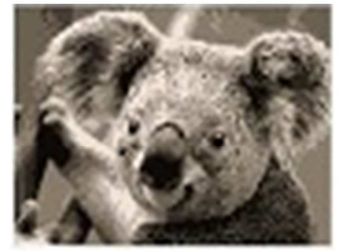

K-means Algorithm

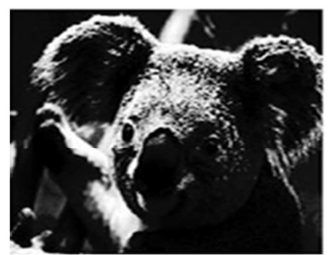

Proposed Algorithm

Figure 5. Original Image, $k$-means Algorithm, proposed Algorithm.

We have taken a different value of $r$ and according to the value of $r$ we get a different result as observed from the figure. So, we need to be careful while taking the value of ra. And we can tune the output result by varying the value of ra. And we can tune the output by varying the value of the sphere penalty radius, $\mathrm{rb}$.

And lastly, we have checked the result in some other images and the segmented output result are shown above in Figure 5. From the figure, we observed that the output result using the proposed algorithm has better segmentation result as compared to the classical k-means algorithm.
The quality of the segmented image is analyzed using the measurement value of Mean Square Error (MSE) [35] and Peak to Signal Noise Ratio (PSNR) [36].

Mean Square Error: It has been used as a standard performance measurement of the output image. It gives how much output image has deviated from the input image.

$$
M S E=\frac{\sum_{N, M}[I 1(m, n)-I 2(m, n)]^{2}}{m * n}
$$

Peak to Signal Noise Ratio: The peak to signal noise ratio is the proportion between maximum attainable powers and the 
corrupting noise that influence likeness of the image. PSNR used to measure the quality of the output image.

$$
S N R=10 \log _{10} \frac{R^{2}}{M S E}
$$

Where $\mathrm{I} 1(\mathrm{x}, \mathrm{y})$ is the input image and $\mathrm{I} 2(\mathrm{x}, \mathrm{y})$ is the segmented image. And the smaller value of MSE means the image is of good quality and the smaller value of PSNR means the image of poor quality. The value of MSE and PSNR of the segmented image is given below in Table 1.

The MSE and PSNR value is calculated for the classical K-means Algorithm as well as the proposed method. The values of MSE are getting very low and the values of PSNR are getting high and when both methods are compared it is found that the proposed method has a better result. We can conclude the output image resulted from the proposed algorithm are of good quality.

Table 1. MSE AND PSNR Values.

\begin{tabular}{lllll}
\hline IMAGE & MSE (proposed algorithm) & PSNR (proposed algorithm) & MSE K-means algorithm & PSNR (K-means algorithm) \\
\hline IMG1 & 2.5579 & 4.0520 & 2.6134 & 3.9587 \\
IMG2 & 1.9875 & 5.1477 & 2.6236 & 3.9418 \\
IMG3 & 1.0441 & 7.9433 & 9.7946 & 8.2210 \\
IMG4 & 2.2544 & 4.6005 & 2.1885 & 4.7293 \\
\hline
\end{tabular}

\section{Conclusion}

We have segmented an image by using a k-clustering algorithm, using the Gaussian Mixture Model cluster to generate the initial centroid. At the same time, partial contrast stretching is used to improve the quality of the original image. And the final segmented result is comparing with the k-means clustering algorithm and we can conclude that the proposed clustering algorithm has better segmentation. The output images are also tuned by varying the hyper sphere cluster radius and we can conclude from that result that by varying the hyper sphere cluster radius we can get different output. And so, we should take the value of the hyper sphere cluster very carefully. Finally, MSE and PSNR are checked and discovered that they have small and large value respective, which are the condition for good image segmentation quality. And comparison for MSE and PSNR are done for the proposed method and classical K-means algorithm and it is found that the proposed method has better performance result. In the future, we can improve the quality of the output image more by using other methods. We can also implement different clustering method using Gaussian Mixture Model clustering algorithm. And lastly, we will implement and analyze in several areas of image segmentation.

\section{References}

[1] Linda G. Shapiro and George C. Stockman (2001):"Computer Vision”, pp 279-325, New Jersey, Prentice-Hall, ISBN 0-13-030796-3.

[2] Barghout, Lauren, and Lawrence W. Lee. "Perceptual information processing system." Paravue Inc. U.S. Patent Application 10/618, 543, filed July 11, 2003.

[3] M. R. Khokher, A. Ghafoor and A. M. Siddiqui, "Image segmentation using multilevel graph cuts and graph development using fuzzy rule-based system", IET image processing, 2012.

[4] V. Dey, Y. Zhang and M. Zhong, "a review on image segmentation techniques with Remote sensing perspective",
ISPRS, Vienna, Austria, Vol. XXXVIII, July 2010.

[5] F. C. Monteiro and A. Campilho, "Watershed framework to region-based image segmentation," in Proc. International Conference on Pattern Recognition, ICPR 19th, pp. 1-4, 2008.

[6] M. Hameed, M. Sharif, M. Raza, S. W. Haider, and M. Iqbal, "Framework for the comparison of classifiers for medical image segmentation with transform and moment based features," Research Journal of Recent Sciences, vol. 2277, p. 2502,2012

[7] R. Patil and K. Jondhale, "Edge based technique to estimate number of clusters in k-means color image segmentation," in Proc. 3rd IEEE International Conference on Computer Science and Information Technology (ICCSIT), pp. 117-121, 2010.

[8] W. Cui and Y. Zhang, "Graph based multispectral high resolution image segmentation," in Proc. International Conference on Multimedia Technology (ICMT), pp. 1-5, 2010.

[9] A. Fabijanska, "Variance filter for edge detection and edge-based image segmentation," in Proc. International Conference on Perspective Technologies and Methods in MEMS Design (MEMSTECH), pp. 151-154, 2011.

[10] M. J. Islam, S. Basalamah, M. Ahmadi, and M. A. S. hmed, "Capsule image segmentation in pharmaceutical applications using edge-based techniques," IEEE International Conference on Electro/Information Technology (EIT), pp. 1-5, 2011.

[11] L. Yucheng and L. Yubin, "An algorithm of image segmentation based on fuzzy mathematical morphology," in International Forum on Information Technology and Applications, IFITA'09, pp. 517-520, 2009.

[12] W. Haider, M. Sharif, and M. Raza, "Achieving accuracy in early stage tumor identification systems based on image segmentation and 3D structure analysis," Computer Engineering and Intelligent Systems, vol. 2, pp. 96-102, 2011.

[13] M. S. A. Shahzad, M. Raza, and K. Hussain, "Enhanced watershed image processing segmentation," Journal of Information \& Communication Technology, vol. 2, pp. 1-9, 2008.

[14] S. Kobashi and J. K. Udupa, "Fuzzy object model based fuzzy connectedness image segmentation of newborn brain MR images," in Proc. IEEE International Conference on Systems, Man, and Cybernetics (SMC), pp. 1422-1427, 2012. 
[15] R. Samet, S. E. Amrahov, and A. H. Ziroglu, "Fuzzy rule-based image segmentation technique for rock thin section images," in Proc. 3rd International Conference on Image Processing Theory, Tools and Applications (IPTA), pp. 402-406, 2012.

[16] M. R. Khokher, A. Ghafoor, and A. M. Siddiqui,"Image segmentation using fuzzy rule based system and graph cuts," in Proc. 12th International Conference on Control Automation Robotics \& Vision (ICARCV), pp. 1148-1153, 2012.

[17] M. Sharif, S. Mohsin, M. J. Jamal, and M. Raza, "Illumination normalization preprocessing for face recognition," in Proc. International Conference on Environmental Science and Information Application Technology (ESIAT), pp. 44-47, 2010.

[18] J. Xiao, B. Yi, L. Xu, and H. Xie, "An image segmentation algorithm based on level set using discontinue PDE," in Proc. First International Conference on Intelligent Networks and Intelligent Systems, ICINIS'08., pp. 503-506, 2008.

[19] F. Zhang, S. Guo, and X. Qian, "Segmentation for finger vein image based on PDEs denoising," in Proc. 3rd International Conference on Biomedical Engineering and Informatics (BMEI), pp. 531-535, 2010.

[20] C. Yuan and S. Liang, "Segmentation of color image based on partial differential equations," in Proc. Fourth International Symposium on Computational Intelligence and Design (ISCID), pp. 238-240, 2011

[21] W. Zhao, J. Zhang, P. Li, and Y. Li, "Study of image segmentation algorithm based on textural features and neural network," in International Conferenceon Intelligent Computing and Cognitive Informatics (ICICCI), pp. 300-303, 2010.

[22] M. Sharif, M. Y. Javed, and S. Mohsin, "Face recognition based on facial features," Research Journal of Applied Sciences, Engineering and Technology, vol. 4, pp. 2879-2886, 2012.

[23] M. Yasmin, M. Sharif, and S. Mohsin, "Neural networks in medical imaging applications: A survey," World Applied Sciences Journal, vol. 22, pp. 85-96, 2013.

[24] L. Zhang and X. Deng, "The research of image segmentation based on improved neural network algorithm," in Proc. Sixth International Conference on Semantics Knowledge and Grid (SKG), pp. 395-397, 2010.

[25] S. A. Ahmed, S. Dey, and K. K. Sarma, "Image texture classification using Artificial Neural Network (ANN)," in Proc. 2nd National Conference on Emerging Trends and Applications in Computer Science (NCETACS), pp. 1-4, 2011.
[26] M. Sharif, M. Raza, S. Mohsin, and J. H. Shah, "Microscopic feature extraction method," Int. J. Advanced Networking and Applications, vol. 4, pp. 1700-1703, 2013.

[27] I. Irum, M. Raza, and M. Sharif, "Morphological techniques for medical images: A review," Research Journal of Applied Sciences, vol. 4, 2012.

[28] S. Zhu, X. Xia, Q. Zhang, and K. Belloulata, "An image segmentation Proc. Third International IEEE Conference on Signal-Image Technologies and Internet-Based System, SITIS'0., pp. 673-678, 2007.

[29] A. Xu, L. Wang, S. Feng, and Y. Qu, "Threshold-based level set method of image segmentation," in Proc. 3rd International Conference on Intelligent Networks and Intelligent Systems (ICINIS), pp. 703-706, 2010.

[30] M. Yasmin, M. Sharif, S. Masood, M. Raza, and S. Mohsin, "Brain image enhancement-A survey," World Applied Sciences Journal, vol. 17, pp. 1192-1204, 2012.

[31] W. Kaihua and B. Tao, "Optimal threshold image segmentation method based on genetic algorithm in wheel set online measurement," in Proc. Third International Conference on Measuring Technology and Mechatronics Automation (ICMTMA), pp. 799-802, 2011.

[32] F. Jiang, M. R. Frater, and M. Pickering, "Threshold-based image segmentation through an improved particle swarm optimisation," in Proc. International Conference on Digital Image Computing Techniques and Applications (DICTA), pp. $1-5,2012$.

[33] D. Barbosa, T. Dietenbeck, J. Schaerer, J. D'hooge, D. Friboulet, and O. Bernard, "B-spline explicit active surfaces: An efficient framework for real-time 3-D region-based segmentation," IEEE Transactions on Image Processing, vol. 21, pp. 241-251, 2012.

[34] A. N. Aimi Salihah, M. Y. Mashor, N. H. Harun and H. Rosline, Colour Image Enhancement Technique for Acute Leukaemia Blood Cell Morphological Feature, In IEEE International Conference on System, Man and Cybernatic, pp. 3677-3682, (2010).

[35] Moreno et al. 2013 Towards no-reference of peak signal to noise ratio estimation based on chromatic induction model International Journal of Advanced Computer Science and Applications.

[36] Wang, Zhou, Bovik and Alan C 2006 Modern image quality assessment (USA: Morgan \& Claypool). 